TRANSACTIONS OF THE

AMERICAN MATHEMATICAL SOCIETY

Volume 348, Number 6, June 1996

\title{
THE STRUCTURE AND ENUMERATION OF LINK PROJECTIONS
}

\author{
MARTIN BRIDGEMAN
}

\begin{abstract}
We define a decomposition of link projections whose pieces we call atoroidal graphs. We describe a surgery operation on these graphs and show that all atoroidal graphs can be generated by performing surgery repeatedly on a family of well-known link projections. This gives a method of enumerating atoroidal graphs and hence link projections by recomposing the pieces of the decomposition.
\end{abstract}

\section{Introduction}

The problem of enumeration of knots and links has always interested knot theorists. In this paper, we introduce a method of enumerating link projections by first decomposing them into pieces called atoroidal graphs. We define surgery on these atoroidal graphs and show how they can be enumerated by performing surgery on a well-known family of link projections. By recomposing these atoroidal graphs, we can thus enumerate link projections. We have included an enumeration of atoroidal graphs up to 12 crossings at the end of the paper.

A link projection is given by a 4 -valent planar graph $G$. To form a link, we can replace each vertex of $G$ by a crossing. To enumerate links in this way, we must first enumerate link projections. It was Kirkman's success in enumerating link projections, or polyhedra as he called them ([K1],[K2]), that formed the basis of the knot tables of both Tait ([T]) and Little ([L1],[L2]). In [C], Conway introduced a notation which made it possible for him to enumerate knots to 11 crossings and links to 10 crossings in a single afternoon, a task that had previously taken years. In his paper, Conway defined a basic polyhedron to be a polyhedron with no bigon regions and showed that every link is obtained by replacing each vertex of a basic polyhedron by a rational tangle. These basic polyhedra are closely related to the atoroidal graphs defined in this paper and can be enumerated using the enumeration of atoroidal graphs described.

The decomposition of a link projection into atoroidal graphs is achieved by cutting the projection along certain non-trivial curves. We then define surgery on an atoroidal graph, giving a new atoroidal graph with one more vertex. This gives a partial ordering on atoroidal graphs, where $G_{1} \prec G_{2}$ if surgery on $G_{1}$ results in $G_{2}$. We show that a graph is initial if and only if it has no vertices of a given type.

Received by the editors October 15, 1994.

1991 Mathematics Subject Classification. Primary 57M25, 57M15, 05C30, 05C85; Secondary 53A35.

Research at MSRI is supported in part by NSF grant no. DMS-9022140.

(C)1996 American Mathematical Society 

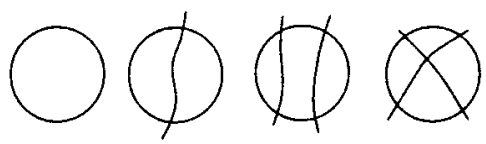

Figure 1. Trivial 0,2,4-curves

Using this fact, we can list all initial objects and thus enumerate atoroidal graphs by repeatedly performing surgery on these initial objects.

The motivation for the paper comes from orbifold theory and hyperbolic geometry, but a background in these is not necessary here. For a reference see [Th]. For readers interested, these aspects are laid out in the section on orbifolds.

I would like to thank Curt McMullen, Joe Christy, Rich Schwartz and especially my advisor Bill Thurston.

\section{Decomposition}

Link Projections. Given a link $L$, a general position projection of $L$ is a 4 -valent graph $G$ embedded in $S^{2}$. As we are only considering such graphs, we will use graph to mean a 4 -valent graph embedded in $S^{2}$.

Let $G$ be a graph with vertex set $V$.

Definition 1. An n-curve of $G$ is a simple closed curve in $S^{2}-V$ transversely intersecting $G$, with $n$ points of intersection.

Let $\alpha$ be an $n$-curve $(n=0,2,4)$ of $G$. Then $\alpha$ splits $S^{2}$ into two disks $D_{1}, D_{2}$. A component $D_{i}$ is called trivial if $D_{i} \cap G$ is either empty, a simple arc, two disjoint simple arcs or two arcs crossing at a single vertex (Figure 1). If $\alpha$ has a trivial component then $\alpha$ is also called trivial. Otherwise $\alpha$ is called non-trivial.

Definition 2. A graph $G$ is irreducible if all $n$-curves $(n=0,2)$ are trivial.

Definition 3. A graph $G$ is atoroidal if all $n$-curves $(n=0,2,4)$ are trivial.

Decomposition. If $\alpha$ is a non-trivial $n$-curve $(n=0,2,4)$ of $G$, then we can decompose $G$ along $\alpha$ into graphs $G_{1}$ and $G_{2}$ as follows. First cut along $\alpha$, this splits the sphere into two disks $D_{1}, D_{2}$. To obtain the graph $G_{i}$ from $D_{i}$, we identify the boundary of $D_{i}$ to a single point. We say that $G$ decomposes into $G_{1}$ and $G_{2}$ along $\alpha$.

We now describe the decomposition of a link projection $G$ into atoroidal graphs. If all $n$-curves $(n=0,2,4)$ in $G$ are trivial then the decomposition is done. Otherwise decompose $G$ into $G_{1}$ and $G_{2}$ along a non-trivial $n$-curve $(n=0,2,4)$, choosing $n$ to be a minimum. Now repeat the decomposition on the resultant graphs $G_{1}$ and $G_{2}$. It is obvious that this decomposition terminates.

The decomposition along non-trivial 0-curves is especially simple, corresponding to splitting a graph into its connected components. Thus both irreducible and atoroidal graphs are connected and to decompose a connected graph, we need only decompose along non-trivial $n$-curves $(n=2,4)$.

\section{Structure of Atoroidal Graphs}

Almost all Atoroidal Graphs are Hyperbolic. To investigate the type of atoroidal graphs possible, we consider the cell division of $S^{2}$ given by $G$ (note 


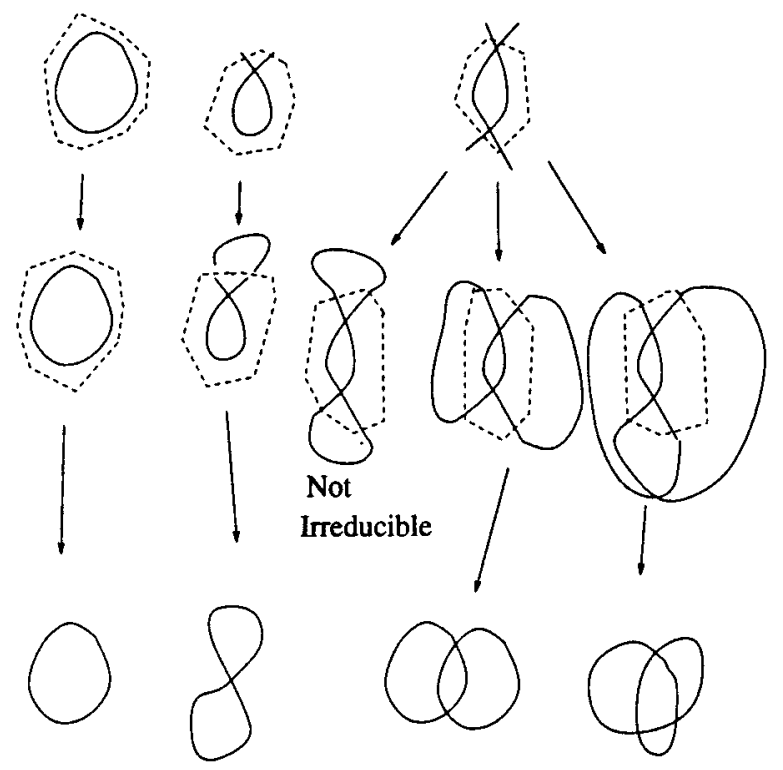

Figure 2. Exceptions

that as $G$ is atoroidal, it is connected). We call the cells, faces of $G$ and a face $F$ is called an $n$-gon if it has $n$ vertices of $G$ on its boundary. If $F$ is an $n$-gon of $G$, then by taking the boundary of a small neighborhood $N_{F}$ of $F$, we get a $2 n$-curve $\alpha_{F}$.

If $G$ has a 0 -gon $F$ then $\alpha_{F}$ is a trivial 0 -curve and therefore it bounds a disk $D$ in $S^{2}-G$. Attaching a disk to the boundary of $N_{F}$ implies that $G$ is a Jordan curve or the unknot projection (Figure 2).

If $G$ has a 1-gon (monogon) $F$ then $\alpha_{F}$ is a trivial 2-curve and therefore bounds a neighborhood of an arc. Attaching a neighborhood of an arc to $N_{F}$ implies that $G$ is a graph having the form of the number eight (Figure 2).

If $G$ has a 2-gon (bigon) $F$ then $\alpha_{F}$ is a trivial 4-curve and therefore bounds either a vertex neighborhood or a neighborhood of two parallel arcs. Attaching a neighborhood of a vertex to $N_{F}$, we get the trefoil projection and attaching a neighborhood of two parallel arcs to $N_{F}$ gives two possible graphs, but only one is also irreducible (Figure 2). Thus $G$ is either the trefoil projection or the Hopf link projection.

Apart from these four exceptional graphs, all other atoroidal graphs have faces that are at least triangular and are called hyperbolic graphs.

Surgery. Given any atoroidal graph $G$ which has a face $F$ with greater than three vertices, we can perform surgery on $G$ to give another atoroidal graph $G^{\prime}$. We choose edges $e, e_{1}, e_{2}$ of $F$, with $e_{1}, e_{2}$ adjacent to $e$. $G^{\prime}$ is obtained by pinching together $e_{1}, e_{2}$, that is, take a simple arc $\alpha \subset F$ with one endpoint in the interior of both $e_{1}$ and $e_{2}$, then shrink $\alpha$ to a single point. The graph $G^{\prime}$ obtained by performing surgery on $G$ has one more vertex than $G$ (Figure 3).

Lemma 1. $G^{\prime}$ is atoroidal. 


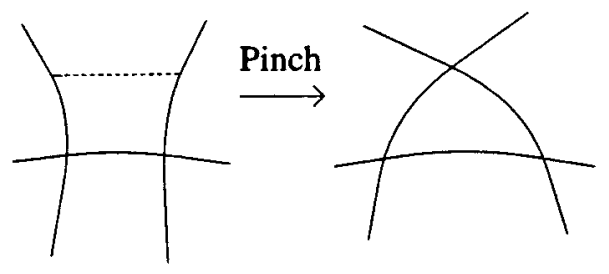

FIGURE 3. Surgery

Proof. Let $\alpha^{\prime}$ be an $n$-curve in $G^{\prime}$ which decomposes $S^{2}$ into disks $D_{1}^{\prime}, D_{2}^{\prime}$. We can get $G$ back by splitting open the new vertex $v$. As this splitting can be done in a small neighborhood of $v$ and $\alpha^{\prime}$ is outside such a neighborhood, we get an $n$-curve $\alpha$ in $G$ which splits $S^{2}$ into disks $D_{1}, D_{2}$. Note that $D_{i}^{\prime}$ and $D_{i}$ are either the same, or the former is obtained from the latter by pinching two edges together.

As $G$ is atoroidal then $\alpha$ is trivial for $n=0,2,4$ and therefore we can assume $D_{1}$ is trivial.

If $n=0,2$ then since $D_{1}$ has at most one edge intersecting it and therefore hasn't enough edges to pinch, $D_{1}^{\prime}=D_{1}$. Therefore $D_{1}^{\prime}$ is trivial.

If $n=4$ then $D_{1}$ is either a vertex neighborhood or a neighborhood of two nonintersecting arcs of $G$. If $D_{1}$ is a vertex neighborhood then the only edges that can be pinched are adjacent, this implies $D_{1}^{\prime}=D_{1}$ and therefore $D_{1}^{\prime}$ is trivial. If $D_{1}$ is a neighborhood of two parallel arcs then either $D_{1}^{\prime}=D_{1}$ or $D_{1}^{\prime}$ is obtained by pinching the parallel arcs of $D_{1}$ together. In the first case $D_{1}^{\prime}$ is automatically trivial and in the second $D_{1}^{\prime}$ is a vertex neighborhood and therefore also trivial.

Thus every $n$-curve in $G^{\prime}(n=0,2,4)$ has a trivial component. Therefore $G^{\prime}$ is atoroidal.

\section{ORBIFOLDS}

This section explains how this work arises out of considering certain orbifolds associated with a link projection. In this setting, the decomposition and surgery we define are the torus decomposition and Dehn surgery on these orbifolds. An orbifold is a generalization of a manifold in which the space is locally modeled on $R^{n}$ modulo the action of a finite group. For example, if a group $G$ acts properly discontinuously on a space $M$, then $M / G$ is an orbifold and is a manifold if the action is also free. For a reference on orbifolds see Chapter 13 of [Th].

Associated Orbifolds. We associate two orbifolds $O_{G}$ and $O_{G}^{\prime}$ to a graph $G$ as follows. We consider $G$ as a graph sitting on $S^{2}$ in $S^{3}$. Let $B$ be a ball in $S^{3}$ with boundary $S^{2}$ and let $V$ be the vertex set of $G$. $O_{G}$ is a polyhedral orbifold with underlying space $X_{O_{G}}=B-V$, singular locus $\Sigma_{O_{G}}=S^{2}-V$ and one-dimensional singular locus $\Sigma_{O_{G}}^{1}=G-V$. The one-dimensional singular locus is marked with $D_{2}$ to indicate that any point on it is modeled by $D^{3} / D_{2}$, where $D_{2}$ acts by two reflections in planes meeting at right angles. $O_{G}^{\prime}$ has underlying space $X_{O_{G}^{\prime}}=S^{3}-V$ and singular locus $\Sigma_{O_{G}^{\prime}}=G-V$. Here the singular locus is one-dimensional and is marked with $Z_{2}$ to indicate that any point on it is modeled on $D^{3} / Z_{2}$, where $Z_{2}$ acts by rotation of order two.

$O_{G}^{\prime}$ is the double of $O_{G}$ in the sense of orbifolds. 
Decomposition and Surgery. In [B], we show that the torus decomposition on the orbifolds $O_{G}$ and $O_{G}^{\prime}$ is the decomposition we've described on $G$. By Andreev's theorem (see [Th]), if $G$ is a hyperbolic graph then $O_{G}$ can be realized as an ideal hyperbolic polyhedron with all dihedral angles right angles. Taking the subgroup of orientation preserving elements of $\pi_{1}\left(O_{G}\right)$ shows us that $O_{G}^{\prime}$ can also be realized as a hyperbolic orbifold.

We show ([B]) that for any graph $G$, the double cover of $O_{G}^{\prime}$ is a link complement denoted by $L_{G}$ with one component for each vertex of $G$. $L_{G}$ is a hyperbolic link complement if and only if $G$ is a hyperbolic graph. Also if $G^{\prime}$ is obtained by surgery on $G$ then the link complement $L_{G^{\prime}}$ is obtained from $L_{G}$ by removing a simple closed curve, which is Dehn drilling on $L_{G}$.

\section{Partial Ordering}

Surgery gives the set of atoroidal graphs a partial ordering $\prec$ by defining $G_{1} \prec G_{2}$ if and only if $G_{2}$ is obtained by performing $r$ successive surgeries on $G_{1}, r=$ $0,1,2, \ldots$ Note that surgery cannot be performed on any of the four exceptional atoroidal graphs and they are never the resultant graph of surgery, therefore they are isolated objects (both initial and final). Thus $\prec$ restricts to a partial ordering on hyperbolic graphs. To study this partial ordering, we first show that the initial objects are a well-known family of graphs. Then by performing surgery on the initial objects, we can generate all atoroidal graphs up to any given vertex number.

After surgery has been performed, we obtain a graph $G$ with a new vertex $v$. The vertex $v$ has the property that it is a vertex of a triangle $T$ and the faces $F_{1}, F_{2}$ meeting at $v$ and adjacent to $T$ are both greater than triangular (Figure 4). A vertex with this local structure we call simple. To find a $\bar{G}$ such that $\bar{G} \prec G$ we might just look for a simple vertex $v$ and cut open at $v$ (there is a unique way to cut open a simple vertex). This doesn't necessarily give an atoroidal graph as the resultant graph may have non-trivial 4-curves (Figure 5). What we will show is that if a graph has a simple vertex $v$ belonging to a triangle $T$, then the graph can be cut open at some vertex of $T$ to give an atoroidal graph. This implies that an initial object cannot have any simple vertices. Before proving the stated result we will use it to show what the initial objects are.

Since the exceptions are isolated, they never arise in a sequence of surgeries and all other initial objects are hyperbolic. Let $G$ be a hyperbolic initial object (not one of the exceptions). Calculating the Euler number of the cell division of $S^{2}$ into faces of $G$, we see that $G$ has a triangular face $T_{1}$. As $G$ is initial, $T_{1}$ has two adjacent triangular faces $T_{2}^{l}, T_{2}^{r}$ (Figure 6). Again using the fact that $G$ is initial,

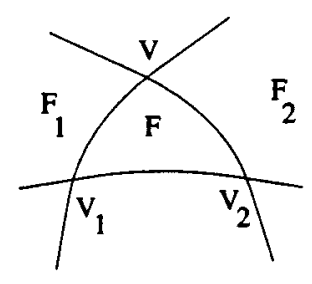

Figure 4. Simple vertex 


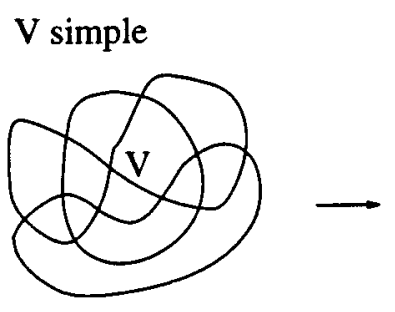

\section{non-trivial}

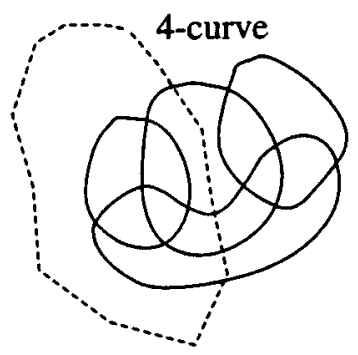

Figure 5. Cutting open at a simple vertex doesn't work

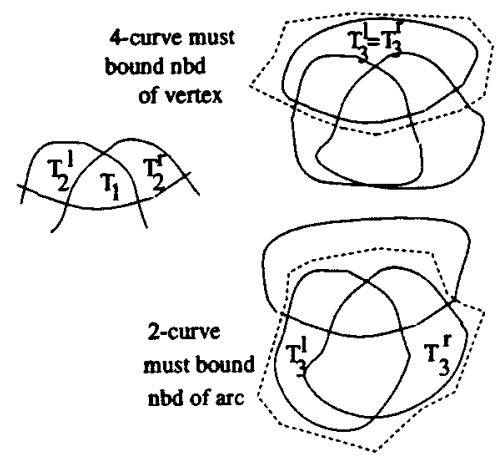

FiguRE 6. Initial setup and the resulting Borromean rings

we have that both $T_{2}^{l}, T_{2}^{r}$ have a neighboring triangular face other than $T_{1}$, labeled $T_{3}^{l}, T_{3}^{r}$ respectively.

If $T_{3}^{l}, T_{3}^{r}$ are disjoint then each has a neighboring triangular face $T_{4}^{l}, T_{4}^{r}$ other than the previous faces $T_{2}^{l}, T_{2}^{r}$. These are unique as the face adjacent to both $T_{3}^{l}$ and $T_{3}^{r}$ is at least four sided. If now $T_{4}^{l}, T_{4}^{r}$ have a common vertex then as before a trivial 2-curve arises. When we close up the loose ends of the 2-curve, we see that $G$ is of the form above (Figure 7 ), which we call $T_{4}$.

If $T_{3}^{l}=T_{3}^{r}$ then taking the boundary of a small neighborhood of the union of the four described triangles, gives a trivial 4-curve. Attaching a trivial disk to this curve, shows that $G$ is the Borromean ring projection. Also if $T_{3}^{l}, T_{3}^{r}$ have a common vertex then similarly we get a trivial 2-curve and again $G$ is the Borromean ring projection, which we call $T_{3}$ (Figure 6).

Continuing this we get the collection of graphs $\left\{T_{n}\right\}_{n \geq 3}$ (Figure 8). These graphs, together with the exceptional atoroidal graphs are the initial objects of $\prec$. The graph $T_{3}$ is the only other isolated object besides the four exceptions. Knowing the initial objects allows us enumerate all atoroidal graphs by performing surgery repeatedly. 


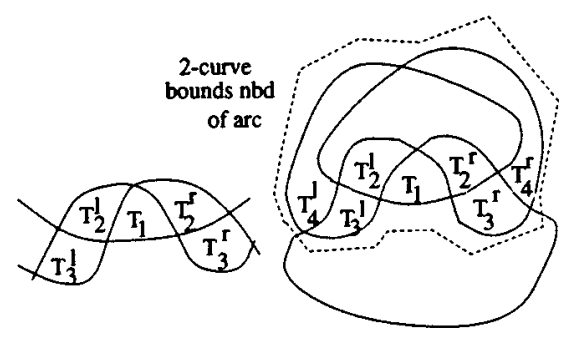

FiguRE 7. Next stage and the resulting graph $T_{4}$

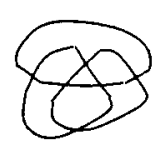

$\mathrm{T}_{3}$

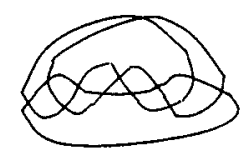

$\mathrm{T}_{6}$

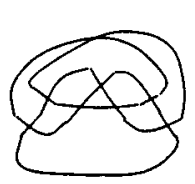

$\mathrm{T}_{4}$

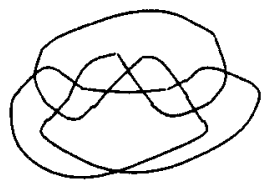

$\mathrm{T}_{5}$

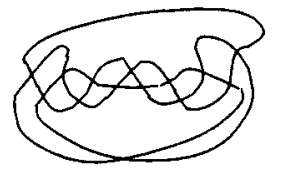

$\mathrm{T}_{7}$

FiguRE 8. $T_{3} T_{4} T_{5} T_{6} T_{7} \cdots$

Lemma 2. Let $G$ be an atoroidal graph with a simple vertex $v$ of triangle $T$ and let $G^{\prime}$ be the graph obtained by cutting $G$ open at $v$. Let $e_{1}^{\prime}, e_{2}^{\prime}$ be the two edges of face $F^{\prime}$ in $G^{\prime}$ pinched to get $G$ then

1. $G^{\prime}$ is irreducible.

2. Any non-trivial 4-curve $\alpha^{\prime}$ of $G^{\prime}$ intersects the face $F^{\prime}$ in a single arc $\beta^{\prime}$ which separates $e_{1}^{\prime}, e_{2}^{\prime}$.

Proof. As above, let $e_{1}^{\prime}, e_{2}^{\prime}$ be the two edges of $F^{\prime}$ pinched together to give $G$ and let $e^{\prime}$ be the edge adjacent to both. If $e^{\prime}$ has a neighborhood $N_{e^{\prime}}$ such that $N_{e^{\prime}} \cap \alpha^{\prime}$ is empty then we can pinch $e_{1}^{\prime}, e_{2}^{\prime}$ in $N_{e^{\prime}}$ with $\alpha^{\prime}$ giving rise to an $n$-curve $\alpha$ in $G$. If $n=0,2,4$ then $\alpha$ is trivial and splits $S^{2}$ into two disks $D_{1}, D_{2}$, with $D_{1}$ trivial. Similarly $\alpha^{\prime}$ splits $S^{2}$ into $D_{1}^{\prime}, D_{2}^{\prime}$ with either $D_{1}^{\prime}=D_{1}$ or $D_{1}^{\prime}$ obtained from $D_{1}$ by cutting open a crossing. In either case, this implies that $D_{1}^{\prime}$ is trivial and therefore $\alpha^{\prime}$ is trivial. Therefore every non-trivial $n$-curve $(n=0,2,4)$ must intersect $F^{\prime}$ in an $\operatorname{arc} \beta^{\prime}$ which has one endpoint on $e^{\prime}$ and the other endpoint on some edge $e_{3}^{\prime}$ of $F^{\prime}$, where $e_{3}^{\prime} \neq e^{\prime}, e_{1}^{\prime}, e_{2}^{\prime}$.

If $n=0$ then $\alpha^{\prime}$ doesn't intersect $G^{\prime}$ and therefore $\alpha^{\prime}$ is trivial.

If $n=2$ then $\alpha^{\prime}$ only intersects $G^{\prime}$ at the two endpoints of $\beta^{\prime}$. If we pinch $e_{1}^{\prime}, e_{2}^{\prime}$ together to get $G$, we can do so by either leaving $e_{1}^{\prime}$ fixed and pulling $e_{2}^{\prime}$ through $\alpha^{\prime}$ or vice-versa. This gives 4-curves $\alpha^{r}, \alpha^{l}$ in $G$ which are identical with $\alpha^{\prime}$ outside a neighborhood of the new vertex $v$ and going either right or left respectively around 


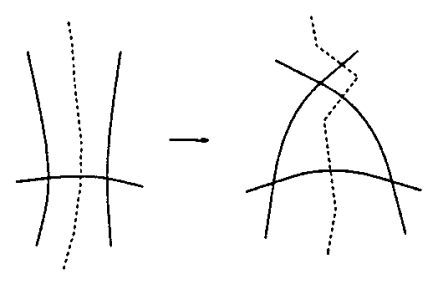

Figure 9. Pinch through $\alpha^{\prime}$
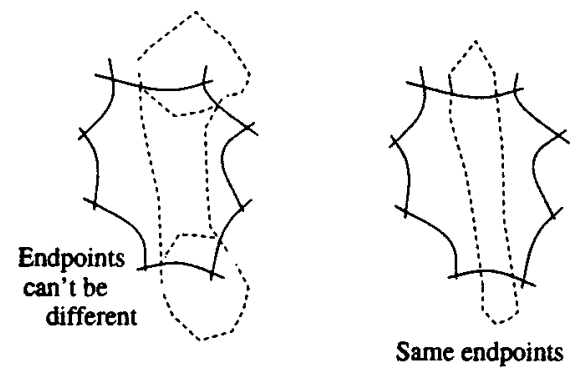

imply trivial

FIgURE 10. Non-trivial curve can't intersect in more than one arc

$v$ as they approach $v$ from inside $T$ (Figure 9). As $G$ is atoroidal, $\alpha^{r}$ is trivial and therefore splits $S^{2}$ into $D_{1}, D_{2}$ with $D_{1}$ trivial. The region containing $v$ also contains another vertex of $T$ and so can't be trivial. Therefore $D_{1}$ contains vertex $v_{2}$ of $T$ and by $G$ being atoroidal, $D_{1}$ must be a neighborhood of $v_{2}$. This implies that $T$ has a neighboring face which is a bigon, contradicting the fact that $v$ is simple. Therefore $G^{\prime}$ has no non-trivial 2-curves and is thus irreducible.

If $n=4$ then $\alpha^{\prime} \cap F^{\prime}$ consists of either one or two arcs. If it is two $\operatorname{arcs} \beta_{1}^{\prime}, \beta_{2}^{\prime}$ then traversing around $\alpha^{\prime}$, we have four connected $\operatorname{arcs} \beta_{1}^{\prime}, \gamma_{1}^{\prime}, \beta_{2}^{\prime}, \gamma_{2}^{\prime}$. We can join the endpoints of $\gamma_{1}^{\prime}$ by another arc $\delta_{1}^{\prime}$ in $F^{\prime}$ such that $\gamma_{1}^{\prime} \cup \delta_{1}^{\prime}$ is a 2-curve in $G^{\prime}$ and therefore trivial. If the endpoints of $\gamma_{1}^{\prime}$ belong to different edges then both components of $S^{2}-\gamma_{1}^{\prime} \cup \delta_{1}^{\prime}$ contain vertices. This contradicts it being a trivial 2-curve. Therefore both $\gamma_{1}^{\prime}, \gamma_{2}^{\prime}$ are contained in adjacent faces to $F^{\prime}$. This implies that $\alpha^{\prime}$ is the boundary of a neighborhood of two parallel arcs of $G^{\prime}$ and is therefore trivial (Figure 10). Therefore any non-trivial 4-curve in $G^{\prime}$ intersects $F^{\prime}$ in a single $\operatorname{arc} \beta^{\prime}$, with $\beta^{\prime}$ separating the two edges in $G^{\prime}$ that we pinch to obtain $G$.

Definition 4. An $n$-curve $\alpha(n \geq 4)$ in $G$ is trivial if and only if either

- $\alpha$ is the boundary of a neighborhood of a vertex of $G$

or

- there exists an arc $\beta$ intersecting $G$ at most once, such that $\beta \cap \alpha=\partial \beta$ and $\partial \beta$ splits $\alpha$ into $\alpha_{1}, \alpha_{2}$ each containing at least two points of $G$.

$\beta$ is called a compression of $\alpha$.

If $G$ is atoroidal then it can be easily shown that the only trivial 6-curves of $G$ are those curves that split $S^{2}$ into two disks, one of which is of the form given above (Figure 11). Disks of this kind are also called trivial. 


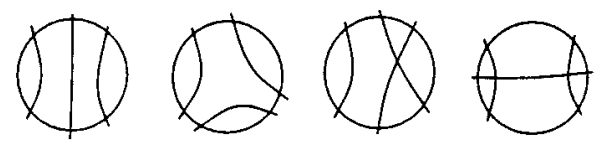

FIGURE 11. Only trivial 6-curves in atoroidal graph

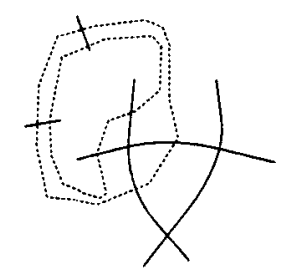

FiguRE 12. All vertices of $T$ are simple

Lemma 3. If $\alpha^{\prime}$ is a non-trivial 4-curve in $G^{\prime}$ then associated with it are two non-trivial 6-curves $\alpha^{l}, \alpha^{r}$ in $G$.

Proof. $\alpha^{\prime}$ intersects $F^{\prime}$ in a single arc $\beta^{\prime}$ separating edges $e_{1}^{\prime}, e_{2}^{\prime}$ and as before we can pinch $e_{1}^{\prime}, e_{2}^{\prime}$ together to get graph $G$. This can be done in two ways, either by fixing $e_{1}^{\prime}$ and pushing $e_{2}^{\prime}$ across $\beta^{\prime}$ or vice-versa. We get two 6-curves $\alpha^{r}, \alpha^{l}$ in $G$, both identical to $\alpha^{\prime}$ outside a neighborhood of the new vertex $v$ and going right, left respectively around the vertex inside the neighborhood of $v$ as before (Figure $9)$.

$\alpha^{r}$ splits $S^{2}$ into disks $D_{1}, D_{2}$ with $D_{1}$ containing vertices $v, v_{1}$ of $T$ and $D_{2}$ containing vertex $v_{2}$. Therefore if $D_{1}$ is trivial then it must be the same type as the fourth disk described (Figure 11). But then $\alpha^{\prime}$ would bound a neighborhood of a vertex of $G$ which contradicts $\alpha^{\prime}$ being non-trivial. If $D_{2}$ is trivial then it is either the same type as the third or fourth disk (Figure 11). If it is the third type then as before $\alpha^{\prime}$ would bound a neighborhood of a vertex of $G$ which contradicts $\alpha^{\prime}$ non-trivial. If it is the fourth type then face $F_{2}$ adjacent to $T$ would be a triangle contradicting $v$ being simple. Therefore $\alpha^{r}$ is non-trivial and similarly $\alpha^{l}$ is non-trivial.

Theorem 1. If $G$ has a simple vertex $v$ of a triangle $T$ then either - splitting open at $v$ gives an atoroidal graph $G^{\prime}$

or

- both other vertices $v_{1}, v_{2}$ of $T$ are simple and splitting at either gives an atoroidal graph.

Proof. If $G^{\prime}$ is not atoroidal then there exists a non-trivial 4-curve $\alpha^{\prime}$ in $G^{\prime}$ and non-trivial 6-curves $\alpha^{r}, \alpha^{l}$ in $G$. Triangle $T$ has adjacent faces $F_{1}, F_{2}, F_{3}$ with both $F_{1}, F_{2}$ non-triangular, as $v$ is simple. If $F_{3}$ is triangular then $\alpha^{r}$ splits $F_{3}$ in two, one piece containing just one vertex, say $v_{1}$, and the other containing two. Therefore $\alpha^{r}$ takes a clockwise path about $v_{1}$ from $T$ through $F_{3}$. If instead we take an anti-clockwise path, $\alpha^{\prime}$ gives us another 4 -curve in $G^{\prime}$, called $\bar{\alpha}^{\prime}$ (Figure 12). Since $\bar{\alpha}^{\prime}$ doesn't intersect $e^{\prime}$, it is trivial. Therefore $\bar{\alpha}^{\prime}$ splits $S^{2}$ into disks $\bar{D}_{1}^{\prime}, \bar{D}_{2}^{\prime}$ with $\bar{D}_{1}^{\prime}$ trivial. $\alpha^{\prime}$ splits $S^{2}$ into $D_{1}^{\prime}, D_{2}^{\prime}$ with $D_{1}^{\prime}$ obtained from $\bar{D}_{1}^{\prime}$ by crossing two 


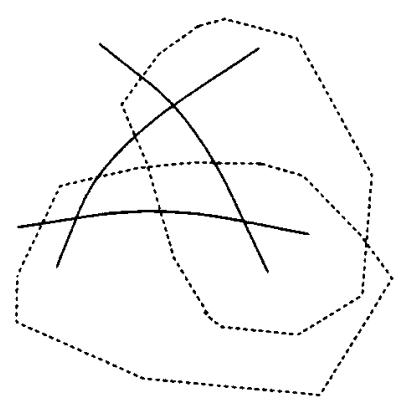

Figure 13. Two intersecting non-trivial 6-curves

adjacent ends. Therefore if $\bar{D}_{1}^{\prime}$ is neighborhood of two parallel arcs then $D_{1}^{\prime}$ is a vertex neighborhood and if $\bar{D}_{1}^{\prime}$ is a vertex neighborhood then $G^{\prime}$ would contain a bigon. Therefore $F_{3}$ must be non-triangular and both vertices $v_{1}, v_{2}$ are simple.

If splitting at $v_{1}$ doesn't give an atoroidal graph then there is a non-trivial 4curve $\alpha_{1}^{\prime}$ in $G_{1}^{\prime}$ and non-trivial 6-curves $\alpha_{1}^{r}, \alpha_{1}^{l}$ in $G$. Consider now the 6-curves $\alpha, \alpha_{1}$ where $\alpha=\alpha^{l}$ and $\alpha_{1}=\alpha_{1}^{r}$. We will show that they can be isotoped to intersect in only two points. Then by showing that they cannot intersect in the given way (Figure 13), the theorem is proven.

First we will show that $\alpha, \alpha_{1}$ can be isotoped to intersect only twice. If they intersect any more than twice, then $S^{2}-\alpha \cup \alpha_{1}$ contains at least four regions that are disks with boundary consisting of one arc of $\alpha$ and one arc of $\alpha_{1}$ and with no two disks having a common boundary arc. As $\alpha \cup \alpha_{1}$ has 12 intersections with $G$, at least one region has boundary being an $n$-curve with $n<4$. Let $D$ be such a region and let $\gamma=\partial D$. Then $\gamma$ is either a trivial 0 or 2-curve, as $G$ is atoroidal. Also $\gamma=\beta \cup \beta_{1}$, where $\beta, \beta_{1}$ are arcs of $\alpha, \alpha_{1}$ respectively. If $\gamma$ is a 0 -curve then either $D$ or $D^{c}$ is a trivial disk. If $D$ is trivial then by an isotopy which pulls $\beta$ through $\beta_{1}$, we can remove two intersections of $\alpha$ and $\alpha_{1}$. If $D^{c}$ is trivial then any of the other three disks with boundary consisting of one arc of $\alpha$ and $\alpha_{1}$ are trivial and hence we can reduce the number of intersections as in first case.

If $\gamma$ is a 2 -curve then both $\beta, \beta_{1}$ intersect $G$, since if $\beta$ didn't then it would be a compression for $\alpha_{1}$, which contradicts $\alpha_{1}$ being non-trivial. Therefore either $D$ or $D^{c}$ is trivial and is therefore a neighborhood of an $\operatorname{arc}$ of $G$. If $D$ is trivial then we can isotope by pulling $\beta$ through $\beta_{1}$, reducing number of intersections of $\alpha$ and $\alpha_{1}$. If $D^{c}$ is trivial then the other three disks with boundary consisting of one arc of $\alpha$ and $\alpha_{1}$ cannot have boundaries being $n$-curves $n \geq 4$, since they can have a maximum of 10 intersections with $G$ between them. Therefore there is region $\bar{D}$, which either doesn't intersect $G$ and thus we can isotope as before to reduce the number of intersections of $\alpha$ and $\alpha_{1}$, or, is a neighborhood of an $\operatorname{arc}$ of $G$, which we can also isotope as before.

Therefore we can assume that $\alpha$ and $\alpha_{1}$ intersect twice and divide $S^{2}$ into four disks. We label these disks $D_{i}, i=1, \ldots, 4$, where $D_{1}, D_{2}, D_{3}$ contain $v_{1}, v_{2}, v$ respectively and $\gamma^{i}=\partial D_{i} . \gamma^{i}$ is an $n_{i}$-curve where $\sum n_{i}=24$ and $n_{i} \geq 4$. Also $\gamma^{i}=\beta^{i} \cup \beta_{1}^{i}$ where $\beta^{i}, \beta_{1}^{i}$ are arcs of $\alpha, \alpha_{1}$ respectively (Figure 13). Note that the $\operatorname{arcs} \beta^{i}, \beta_{1}^{i}$ have duplication, with each of the arcs of $\alpha$ and $\alpha_{1}$ repeated twice, as each arc forms part of the boundary of a region on both sides. For ease of labeling 

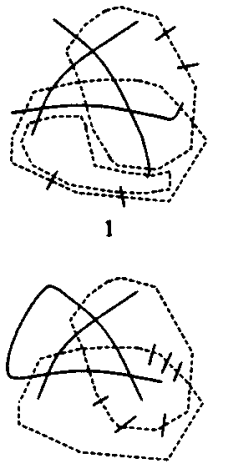
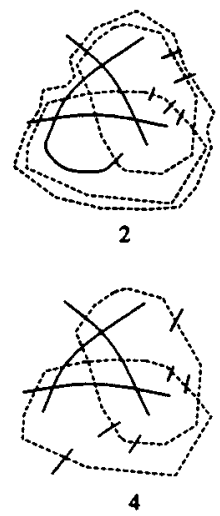

Figure 14. Cases

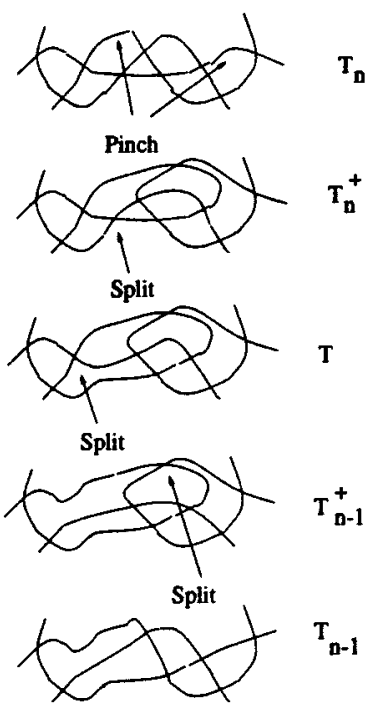

FIGURE 15. $T_{n-1}^{+} \prec T_{n}^{+}$

we will leave this duplication, considering the two labels that have the same arc to correspond to the two sides of the arc on $\alpha$ or $\alpha_{1}$.

Case 1:If $n_{2}=4$ then $D_{2}$ is a neighborhood of $v_{2}$ and both $\beta^{2}$ and $\beta_{1}^{2}$ intersect $G$ twice, otherwise we get a compression of $\alpha$ or $\alpha_{1}$. Therefore $\gamma^{1}$ is a 6-curve and inside $D_{1}$ is a 4-curve $\tilde{\gamma}^{1}$ (Figure 14). If it is the boundary of a neighborhood of two parallel arcs of $G$ then this implies that either $F_{3}$ is a is a bigon or $\alpha_{1}$ is a trivial 6 -curve. If it is the boundary of a vertex neighborhood then this would imply that $F_{3}$ is triangular. Therefore $n_{2} \neq 4$.

Case 2:If $n_{1}=4$ then $\beta_{1}^{1}$ intersects $G$ only twice, otherwise $\beta^{1}$ is a compression of $\alpha_{1}$. Therefore $\gamma^{4}$ is a 6 -curve and $D_{4}$ contains a 4-curve $\tilde{\gamma}^{4}$ (Figure 14). If it is the boundary of a neighborhood of two parallel $\operatorname{arcs}$ of $G$ then this implies that either $F_{1}$ is a bigon or $\alpha$ is a trivial 6 -curve. If it is the boundary of a vertex 
neighborhood then this implies that $F_{1}$ is triangular. Therefore $n_{1} \neq 4$ and by symmetry $n_{3} \neq 4$.

Case 3:If $n_{4}=4$ then $D_{4}$ must be a neighborhood of parallel $\operatorname{arcs}$ of $G$, which implies $F_{1}$ is a bigon (Figure 14). Therefore $n_{4} \neq 4$.

Case 4:If $n_{i}=6$ then each arc $\beta^{i}, \beta_{1}^{i}$ intersects $G$ exactly three times. Therefore $\tilde{\gamma}^{1}, \tilde{\gamma}^{4}$ are both 4-curves. If $\tilde{\gamma}^{4}$ is the boundary of a neighborhood of two parallel arcs of $G$ then this implies that either $F_{1}$ is a bigon or both $\alpha$ and $\alpha_{1}$ have compressions, contradicting the fact that $F_{1}$ is not a bigon and both $\alpha, \alpha_{1}$ are non-trivial (Figure 14). If $\tilde{\gamma}^{4}$ is the boundary of a vertex neighborhood then this implies that $F_{1}$ is triangular, another contradiction. Therefore the case of $n_{i}=6$ is ruled out.

Having ruled out all possible cases, we have shown that 6 -curves cannot intersect in the way that $\alpha$ and $\alpha_{1}$ would have to if both existed. This implies that if $\alpha$ exists then $\alpha_{1}$ can't exist. Therefore if $G^{\prime}$ is not atoroidal then $G_{1}^{\prime}$ is atoroidal. Similarly, if $G^{\prime}$ is not atoroidal then $G_{2}^{\prime}$ is atoroidal.

We have shown that the initial objects of $\prec$ are $\left\{T_{n}\right\}_{n>3}$ along with the four exceptions. $T_{n}$ is the projection of the $(3, n)$ torus link with the link having three components if three divides $n$ and having one component otherwise. From this, we see that $T_{n}$ has symmetries taking any directed edge of one of the non-triangular faces to any other. Therefore any surgery on $T_{n}$ gives the same graph, which we call $T_{n}^{+}$.

Lemma 4. If $T_{n} \prec G$ and $T_{n} \neq G(n>4)$ then $T_{n-1} \prec G$.

Proof. If $T_{n} \prec G$ and $T_{n} \neq G$ then $T_{n}^{+} \prec G$. As before (Figure 7 ), we can pinch together edges of $T_{2}^{l}$ and $T_{4}^{r}$ to get $T_{n}^{+}$. Only one vertex of $T_{1}$ is simple, so we cut open at that vertex first. This reduces the pinched $T_{2}^{l}$ to a triangle which has only one simple vertex. After we cut open this simple vertex (Figure 15), the resulting graph is $T_{n-1}^{+}$. Therefore we have that $T_{n-1} \prec T_{n-1}^{+} \prec T_{n}^{+} \prec G$.

Therefore if $C_{n}$ is the set of proper descendants of $T_{n}$, that is, the set of all descendants except $T_{n}$, then

$$
C_{4} \supseteq C_{5} \supseteq C_{6} \supseteq \cdots \supseteq C_{n} \cdots
$$

\section{ENUMERATion}

We now have a way to enumerate atoroidal graphs up to any prescribed crossing number by performing surgery on the initial objects. Figure 16 is the enumeration of atoroidal graphs up to twelve crossings. To enumerate prime link projections we need only recombine the atoroidal graphs as follows. We choose two atoroidal graphs $G_{1}$ and $G_{2}$ with vertices $v_{1}$ and $v_{2}$ respectively. Now take the complement of a neighborhood of each vertex and attach their boundaries, making sure to match up the strands of the graphs. In recombining we do not use the first three exceptions as either they have no vertices or the complement of a neighborhood of a vertex is trivial. To enumerate the basic polyhedra of Conway the trefoil projection is also not used as the complement of a vertex is a bigon. 
o
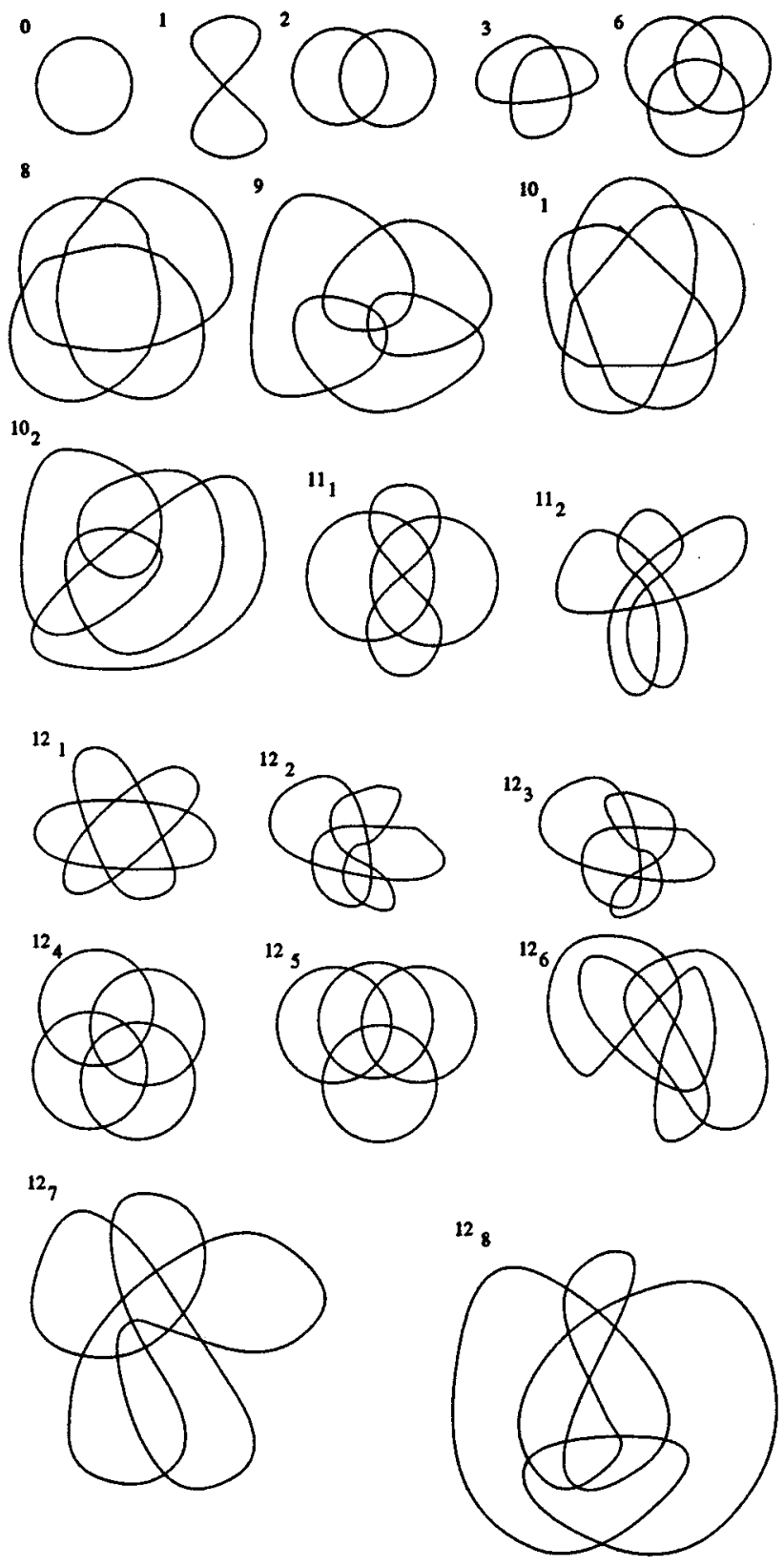

Figure 16. Atoroidal graphs of twelve crossings or less 


\section{REFERENCES}

[B] M. Bridgeman, Volume Increase under Dehn Drilling Operations, Phd. thesis, Princeton, June 1994.

[C] J. H. Conway, An enumeration of knots and links, and some of their algebraic properties, Computational Problems in Abstract Algebra, Pergamon, Oxford, (1970). MR 41:2661

[K1] T. P. Kirkman, The enumeration, description and construction of knots of fewer than ten crossings, Trans. Roy. Soc. Edinburgh, 32 (1885).

[K2] T. P. Kirkman, The 364 unifilar knots of ten crossings enumerated and defined, Trans. Roy. Soc. Edinburgh, 32 (1885).

[L1] C. N. Little, Non-alternate \pm knots, of order eight and nine, Trans. Roy. Soc. Edinburgh, 35 (1889).

[L2] C. N. Little, Alternate \pm knots of order 11, Trans. Roy. Soc. Edinburgh, 36 (1890).

[T] P. G. Tait, On knots I, II, III (1887, 1884, 1885), Scientific Papers I.

[Th] W. P. Thurston, The geometry and topology of three manifolds, Princeton Lecture Notes, (1979).

Mathematical Sciences Research Institute, 1000 Centennial Drive, Berkeley, CaliFORNIA 94720

Current address: Department of Mathematics, Loyola University, New Orleans, Louisiana 70118

E-mail address: bridgemn@beta.loyno.edu 